\title{
Imaging and Pathological Features of Alveolar Soft Part Sarcoma: Analysis of 16 Patients
}

\author{
Malvika Gulati ${ }^{1} \quad$ Abhenil Mittal $^{2} \quad$ Adarsh Barwad $^{3} \quad$ Rambha Pandey $^{4}$ Sameer Rastogi ${ }^{2}$ \\ Ekta Dhamija1일
}

${ }^{1}$ Department of Radiodiganosis, Dr. B. R. A. IRCH, All India Institute of Medical Sciences, New Delhi, India

2 Department of Medical Oncology, Dr. B. R. A. IRCH, All India Institute of Medical Sciences, New Delhi, India

${ }^{3}$ Department of Pathology, Dr. B. R. A. IRCH, All India Institute of Medical Sciences, New Delhi, India

${ }^{4}$ Department of Radiation Oncology, Dr. B. R. A. IRCH, All India

Institute of Medical Sciences, New Delhi, India

Address for correspondence Ekta Dhamija, MD, Department of Radiodiagnosis, Dr. B. R. A. IRCH, All India Institute of Medical Sciences, Room Number 137, First Floor, New Delhi 110029, India (e-mail: drektadhamija.aiims@gmail.com).

Indian J Radiol Imaging 2021;31:573-581.

\section{Abstract \\ Keywords \\ - alveolar soft part sarcoma \\ - soft tissue sarcoma \\ - imaging \\ - feeding vessels \\ - metastatic disease}

Context Alveolar soft part sarcoma (ASPS) is a rare soft tissue tumor most commonly occurring in deep intramuscular plane of lower extremities of adolescents and young adults. It is a highly vascular, slow growing tumor with malignant potential having lung as the most common site of metastases at the time of presentation.

Aims The aim is to review the imaging findings of ASPS and determine characteristic imaging features of this rare tumor.

Materials and Methods Sixteen patients having histopathological diagnosis and preoperative imaging of ASPS attending the dedicated sarcoma clinic at our institute were included in the study. The demographic, clinical, and imaging data were retrieved from the case records and then evaluated for characteristic imaging features which may raise suspicion of ASPS.

Results The patients ranged from 3 to 72 years of age and with a slight male preponderance. Of the eight CECTs evaluated, 62.5\% tumors showed well-defined lobulated margins, $87.5 \%$ cases showed intense enhancement with presence of feeder vessels. On CEMRI of 10 patients, $70 \%$ had well circumscribed lobulated margins with intense enhancement and tortuous flow voids in most of them. All cases showed T2 hyperintense signal. Fourteen of 16 (87.5\%) patients had metastatic disease with lung as the most common site (92.8\%).

Conclusion ASPS is a rare soft tissue sarcoma seen in children and young adults. Imaging may mimic a vascular malformation due to the presence of tortuous feeders. Misdiagnosis at an early stage may lead to later metastatic presentation of the disease, thus emphasizing the need to suspect it on imaging.
DOI https://doi.org/ 10.1055/s-0041-1735501. ISSN 0971-3026. (c) 2021. Indian Radiological Association. All rights reserved.

This is an open access article published by Thieme under the terms of the Creative Commons Attribution-NonDerivative-NonCommercial-License, permitting copying and reproduction so long as the original work is given appropriate credit. Contents may not be used for commercial purposes, or adapted, remixed, transformed or built upon. (https://creativecommons.org/ licenses/by-nc-nd/4.0/)

Thieme Medical and Scientific Publishers Pvt. Ltd., A-12, 2nd Floor, Sector 2, Noida-201301 UP, India 


\section{Introduction}

Soft tissue sarcomas (STS) are a rare malignancy with undifferentiated pleomorphic sarcoma (previously called malignant fibrous histiocytoma), liposarcoma, and leiomyosarcoma being the most frequent histologies in adults. ${ }^{1}$ Alveolar soft part sarcoma (ASPS) comprises $<1 \%$ of all STS with literature limited to case reports and series. ${ }^{2}$ As per the $5^{\text {th }}$ WHO classification of soft tissue tumors updated in 2020 , ASPS falls under the malignant category of "tumors of uncertain differentiation" ${ }^{3}$ with extremities being the most common site of occurrence. It mostly occurs in children and young adults in the age group of 15 to 35 years. ${ }^{4}$ Nearly two-thirds of adults and one-third of the children have metastases to lungs, lymph nodes, and brain at the time of diagnosis. Metastases are known to occur after long tumor free intervals, approximately $38 \%$ of metastases occurring after 10 years of diagnosis with or without local site recurrence. ${ }^{4,5}$ Patients of ASPS usually have a poor prognosis. The mainstay of treatment remains total surgical excision and metastatectomy as it is unresponsive to conventional chemotherapy. Various targeted immunotherapies have been tried; however, no standard medical treatment protocol exists. $^{6}$

Its typical histopathological appearance is arrangement of large epithelioid tumor cells in a pseudo"alveolar" pattern surrounded by sinusoidal vascular channels thus giving its name. ASPS is characterized by the unbalanced translocation $t(X ; 17)(p 11 ; q 25)$, which creates the ASPSCR1-TFE3 fusion protein, having angiogenic properties. ${ }^{7,8}$

The imaging features often mimic vascular tumors and malformations due to its highly vascular nature and feeders, which may delay appropriate work-up. ${ }^{4,6}$ Further, it requires review by pathologists having expertise in sarcomas and application of special immunohistochemical or genetic markers for exact characterization. Hence, the initial suspicion must be raised on imaging. Contrast-enhanced MRI serves is a major tool in the work-up of soft tissue tumors to suggest the diagnosis and assess resectability. ASPS are usually located in the deep intramuscular compartment of an extremity, demonstrating lobulated well-defined margins showing hyperintense signal on T2WI and intense enhancement. Feeder vessels within and in the periphery of the tumor are typical. ${ }^{4,9-12}$ Metastases most commonly to the lung are present at the time of presentation ${ }^{4,9,10}$. This study was conducted to review and determine the imaging features of ASPS patients visiting dedicated sarcoma clinic of our tertiary care center.

\section{Materials and Methods}

This retrospective study was conducted on patients with histopathological diagnosis of ASPS presenting to dedicated sarcoma clinic of our institute during the last 3 years (2016 to 2019), after obtaining approval from institute's ethics committee, with waiver for informed consent. The demographic, clinical, imaging, and pathological details were obtained from the patients' institutional case records.
Inclusion criteria: Histopathological diagnosis of ASPS in patients presenting to sarcoma clinic, confirmed by sarcoma pathologist of our institute. Of these patients, the ones with availability of at least one baseline cross-sectional imaging (computed tomography [CT] or magnetic resonance imaging [MRI] or positron emission tomography computed tomography [PET-CT]) of the primary tumor and adequate clinical details such as age and gender of the patient were included.

Exclusion criteria: Non availability of baseline CT/MRI/ PET-CT.

Histopathology: The pathological diagnosis of ASPS was established by pathologist (A.B.) with an experience in sarcomas. In the patients, who underwent biopsy or resection outside, the slides were reviewed by the same sarcoma pathologist of our institute, before deciding on treatment plan.

Imaging analysis: Imaging details in the form of ultrasonography (USG), CT, MRI, or PET-CT were obtained from the patients' files. For the patients' whose imaging was available and was done outside, the films were reviewed by the radiologist (E.D.). The imaging features which were recorded, included: size, site, margins, attenuation/ intensity/echogenicity/enhancement pattern/avidity based on the imaging modality, surrounding and internal vascularity, feeders in vicinity, intratumoral fat/necrosis/ calcification, surrounding structural infiltration, distant metastases, and treatment response.

\section{Definitions}

Margins: The mass was categorized to have lobulated or circumscribed margins when the entire mass could be delineated with no infiltration or irregularity along the outline. However, few lobulations could be seen. Infiltrative margins were noted when there was evident involvement of surrounding muscles, bones or vessels at the site of irregular margins.

Enhancement characteristics were classified as intense, moderate, no or minimally enhancing as compared with surrounding muscles and vessels.

Internal imaging parameters were assessed on CT as iso/hypo/hyperdense and signal characteristics on T1WI and T2WI as equal or iso/hypo/hyperintense as compared with surrounding muscles-that is, same/low/higher than muscles, respectively.

Note was made of presence or absence of intratumoral and peripheral feeder vessels and distant metastases.

PET-CT was evaluated for similar findings as in CT. In addition, the SUV value and presence of any distant metastases $>1 \mathrm{~cm}$ in size were also recorded.

Follow up: All the cases were followed up till February 2020 in the outpatient visits by interviews with the patients themselves or the attendants.

Statistical analysis: The collected data was transformed into variables, coded and entered in Microsoft Excel. Data were analyzed and statistically evaluated using SPSS-PC-17 version.

Quantitative data was expressed in ranges and mean while qualitative data were expressed in percentage. 


\section{Results}

A total of 17 patients of ASPS reported to dedicated sarcoma clinic of our tertiary care center, one was excluded due to nonavailability of imaging. The age ranged from 3 to 72 years (mean $29.2 \pm 15.2$ years) and slight male preponderance (male: female 5:3).

Of these 16 patients, six patients had only MRI, four had only CT, one had MRI and PET-CT, one had MRI and CT, two had only PET-CT, one had USG, PET-CT as well as MRI and one had USG and MRI.

Size and site: The primary tumor size ranges from 3.6 to $17 \mathrm{~cm}$ (mean $8.29 \mathrm{~cm}$ ).

Most common location was extremities (10/16 cases, $62.5 \%$ ) with $80 \%$ (8/10) occurring in lower extremities. Other rare sites included were head and neck $(2 / 16,12.5 \%)$ and chest wall, intrathoracic or intra-abdominal. One patient presented with metastases from ASPS but without a known primary.

Histopathology: All the cases showed typical histomorphology of alveolar STS in the form of epithelioid appearing cells arranged in distinct nesting or organoid pattern with central dyscohesive cells giving appearance of pseudoalveolar pattern (-Fig. 1a). In one of the cases, the tumor was seen predominantly in nesting and solid pattern. Cells were generally monomorphic, polygonal shaped with distinct cytoplasmic boundaries ( $\mathbf{- F i g}$. 1b). The nucleus was round with finely dispersed chromatin with prominent nucleoli in most of them. Occasional mitotic figures were noted. Diastase treated periodic acid Schiff (PAS) stain highlights rhomboid-shaped or rod-shaped intracytoplasmic PAS positive inclusions (-Fig. 1c). All the cases demonstrated nuclear immunoreactivity on immunohistochemistry (IHC) for TFE-3.

Imaging: Two patients had undergone baseline ultrasound which revealed a circumscribed lobulated homogeneously hypoechoic mass showing multiple enlarged tortuous vessels within. Four patients had CECT of the local part and four patients had PET CT, hence a total of eight CTs were evaluated for primary tumor.

On CECT, the primary masses had circumscribed lobulated margins (five of eight patients, 62.5\%), showed intense enhancement on post contrast scans (seven of eight patients, 87.5\%) with multiple feeder vessels within and in the periphery of the tumor (seven of eight patients, 87.5\%). None of the tumor showed necrosis and only one case had the presence of calcification (-Table $\mathbf{1}$ ).

PET CT was available for four patients and the primary sites and metastatic disease was avid with an SUV of $>2.5$.

MRI of 10 patients was evaluated. Most of the patients showed circumscribed lobulated margins (seven-tenths, $70 \%$ ), iso to intermediate signal on T1WI (six-tenths, 60\%) and hyperintense signal on T2WI (ten-tenths, 100\%). Intense or moderate enhancement was seen on gadolinium-enhanced MRI with presence of multiple flow voids in 9 of 10 patients (90\%) (-Table 2 ). The main role of MRI was to assess the resectability of tumor and 7 of 10 cases were found resectable on MRI. Out of these seven, only four patients could undergo surgery as rest had metastatic disease otherwise.

Metastatic disease: Fourteen of 16 (87.5\%) patients had presence of metastases at the time of presentation in our clinic. Most common metastatic site was lung in 13 of 14 patients (92.8\%). Two patients with lung metastases underwent metastatectomy for lung nodules. Next most common site of metastasis was bones $(4 / 14,28.6 \%)$. Five patients had metastases to more than one site which included liver (three), adrenal (one), and brain (two), and lymph nodes (one).

Follow up: Most of the cases were operated outside hospitals. Recurrence post-surgery occurred in six of 16 (37.5\%) patients; revision surgery was done in one and surgical excision with positive margins was obtained in another patient. Medical treatment was given as first line treatment in seven of 16 patients for locally unresectable disease, extensive metastases, or unknown primary tumor.

Till last follow-up all 16 patients were alive and had stable disease.

\section{Discussion}

STS are a rare malignancy accounting for $1 \%$ of all cancers. Under the WHO 5th edition of classification of soft tissue tumors, ASPS is a rare tumor belonging to the malignant

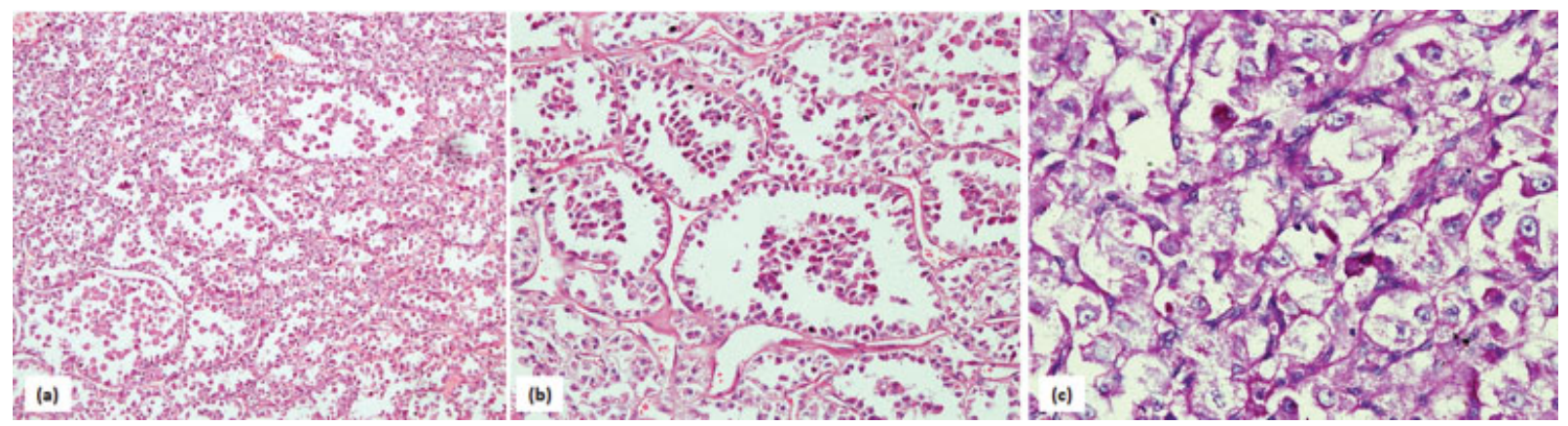

Fig. 1 Histopathology of alveolar soft part sarcoma: (a) Low power picture showing polygonal appearing cells arranged in distinct nesting or organoid pattern with central dyscohesive cells giving appearance of pseudoalveolar arrangement. (b) Cells are generally monomorphic, polygonal shaped with distinct cytoplasmic boundaries. The nucleus is round with finely dispersed chromatin with prominent nucleoli in most of them. Occasional mitotic figures are noted. (c) Diastase treated periodic acid Schiff (PAS) stain highlight rod-shaped intracytoplasmic PAS positive inclusions. 

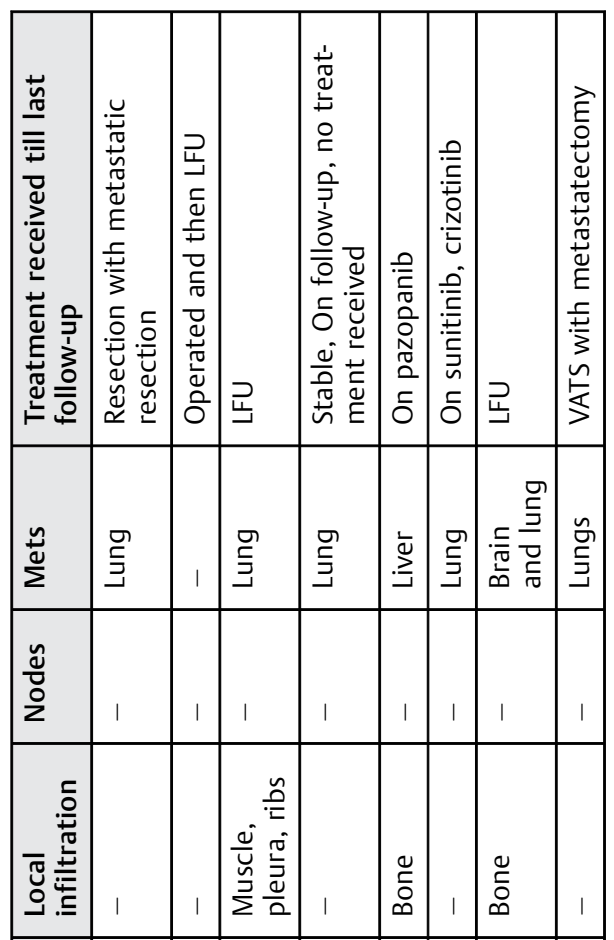

苋

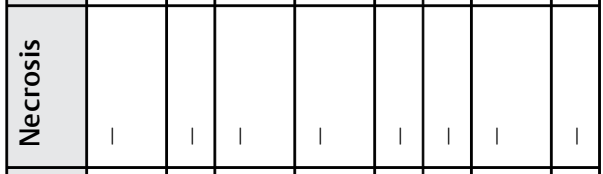

这

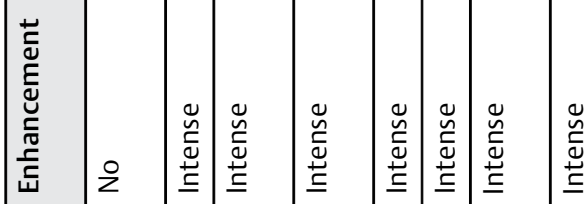

$\overline{\mathrm{E}}$

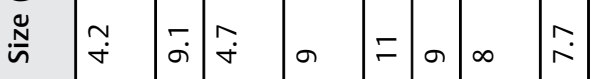

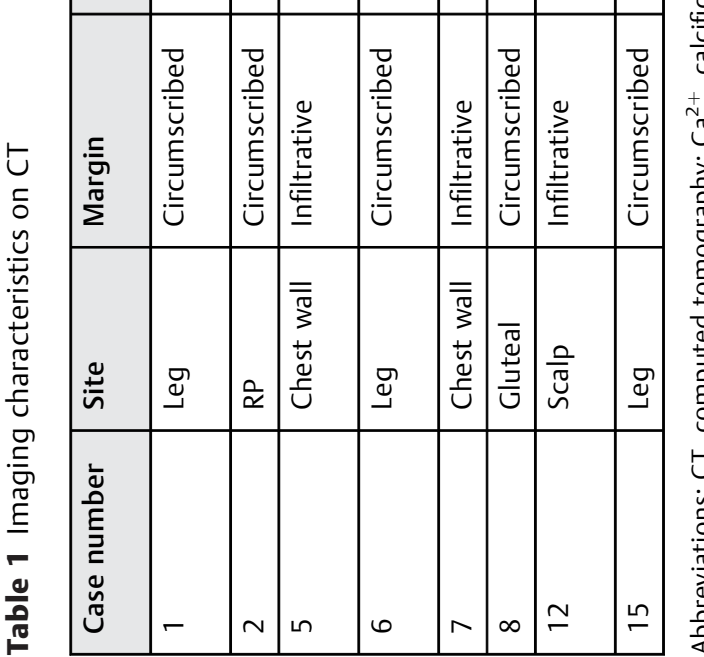

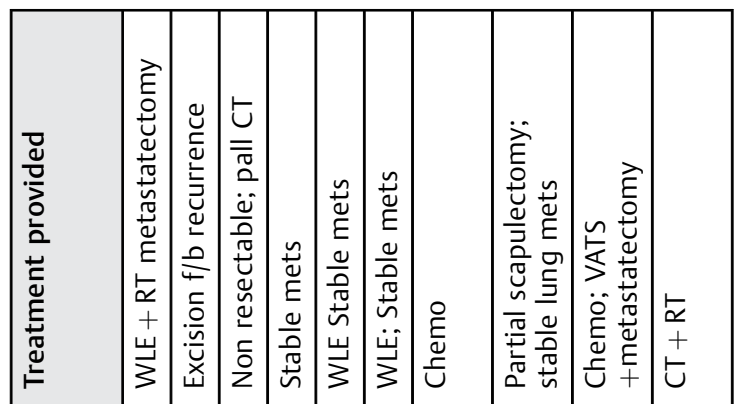

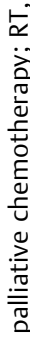

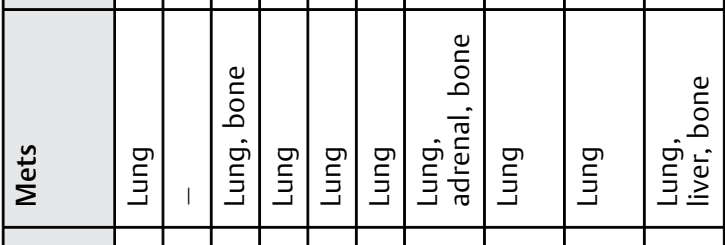

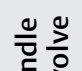

这旁

高

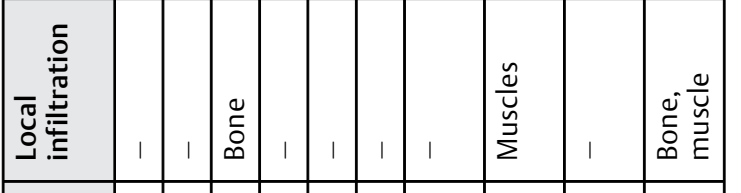

亗

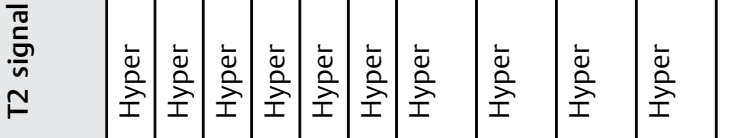

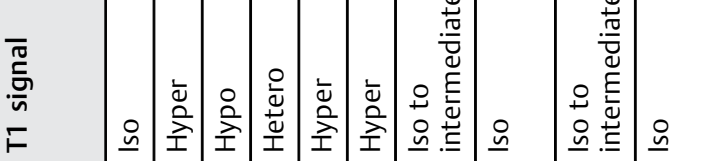

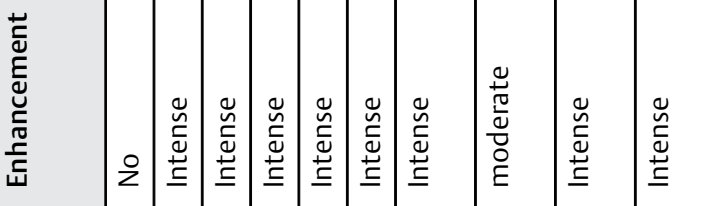

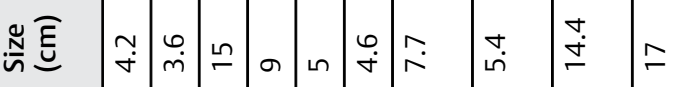

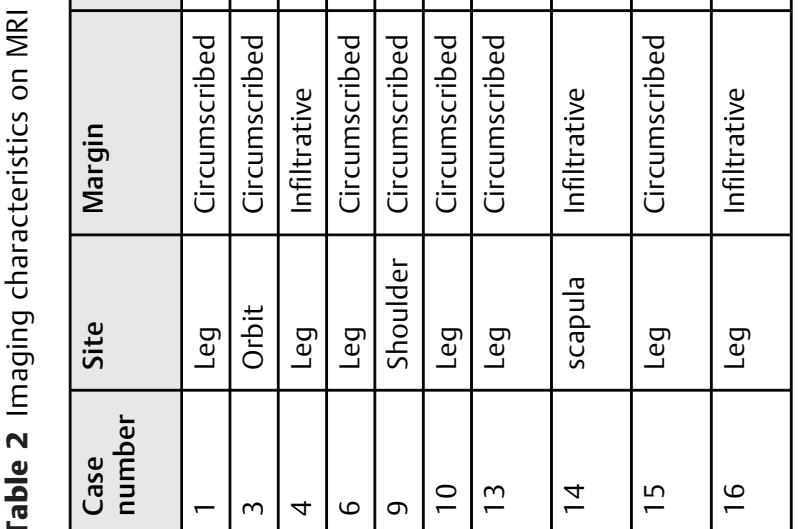

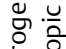
埒 离 过 富蓄 药 ㄴ. 4 䓂 . 商 
group of tumors of uncertain differentiation. ${ }^{3}$ The common occurrence in the skeletal muscle raises speculations of myogenic origin; however, inability to consistently demonstrate IHC markers for the same raises uncertainty of cell of origin. This may be attributed to its origin from myxoid precursors without differentiation. ${ }^{13}$ Histologically, tumor is known to have a homogenous appearance across the mass and between different patients. It has a characteristic appearance of cells arranged in pseudoalveolar or organoid pattern between fibrovascular septae and delicate vessels. In addition, there are intracytoplasmic rod-shaped inclusions which are better highlighted by PAS stain and are diastase resistant. Ultrastructurally, it shows membrane bound rhomboid shaped crystals that are composed of periodic lattice-work of rigid fibrils with dimension of 5 to $7 \mathrm{~nm}$ and periodicity of $10 \mathrm{~nm}$. On IHC, the cells are usually negative for epithelial markers like cytokeratins and EMA, neural markers like glial fibrillary acidic protein, and neuroendocrine markers such as synaptophysin. Nuclear reactivity for MyoD1 is variable. ${ }^{14}$ In addition, most of the cases showed nuclear immunoreactivity for TFE-3 immunostain. An unbalanced translocation $\mathrm{t}(\mathrm{X} ; 17)(\mathrm{p} 11 ; \mathrm{q} 25)$, which creates the ASPSCR1-TFE3 fusion protein is classically seen in these cases. ${ }^{7,8}$ Rarely this translocation may also be found in some pediatric renal cell carcinomas and periendothelial epithelioid cell tumors. ${ }^{10}$ There are reports where histologically renal cell carcinomas mimicked appearance of ASPS due to fascicular arrangement of cells in some areas; however, absence of any renal primary ruled out the diagnosis. ${ }^{10}$ Endothelial and periepithelial tumors can be distinguished as these push intact and uninvolved endothelium. ${ }^{7}$

The common presentation of these tumors is as a slowly growing painless mass. Due to their highly vascular nature and feeder vessels, they are often misdiagnosed as arteriovenous malformations (AVMs). Later, these tumors present with distant metastasis due to the diagnostic delay as histopathological examination or surgery is almost never done in the latter. Pennacchioli et al showed that around $9 \%$ (3/33) of their patients were initially thought to have vascu- lar malformations on MRI, hence delaying the diagnosis. ${ }^{6}$ AVMs usually lack any soft tissue component in contrast to sarcomas; they are hypointense on T1 and T2 and show early arterial feeders and early venous washout. ${ }^{4,6}$ Though conventional angiography was not done in any patient in our study, findings such as arterial feeders with prolonged staining of the tumor and late washout indicate soft tissue vascular tumor rather than an AVM. ${ }^{14}$

Another commonly confused entity with ASPS can be hemangiomas which are benign vascular tumors. In a review by Viry et al of six children with ASPS, one case of orbital ASPS was initially suspected to be a hemangioma and embolized. However, hemangiomas are known to occur in a much younger age group than ASPS and are usually present since birth. These show characteristic involution with age and can often contain a fatty component on imaging. Also, they enhance in the early arterial phases compared with soft tissue tumors which enhance later in the late arterial or early venous phases. ${ }^{4}$

Small case series or reviews with maximum of 25 cases of ASPS are present in literature due to the rarity of the disease. ${ }^{9}$ Most of the cases of this rare tumor occur in children and young adults. The age group of the cases in our study ranged from 3 years to 72 years with the mean age of 29.25 years of age. The mean age reported in other studies ranged from 18.5 to 36 years. ${ }^{9,11}$ These are seen to occur in a much younger age group than other more common sarcomas like liposarcoma, GIST, and leiomyosarcoma which peak in the fifties.

In contrast to available literature, we had more males with ASPS (six females and 10 males); however, due to small sample size, it would not be correct to say that ASPS has specific gender predilection. It is postulated that due to the characteristic translocation involving $X$ chromosome, ASPS is twice more probable to occur in females. $4,9,10$

The most common site of occurrence in our study was found to be extremities ( 10 of 16 cases) which is in concordance with other authors $^{4,9-11}$ (- Figs. 2 and 3). Other tumor locations found in our cohort were retroperitoneum, orbit, chest wall,

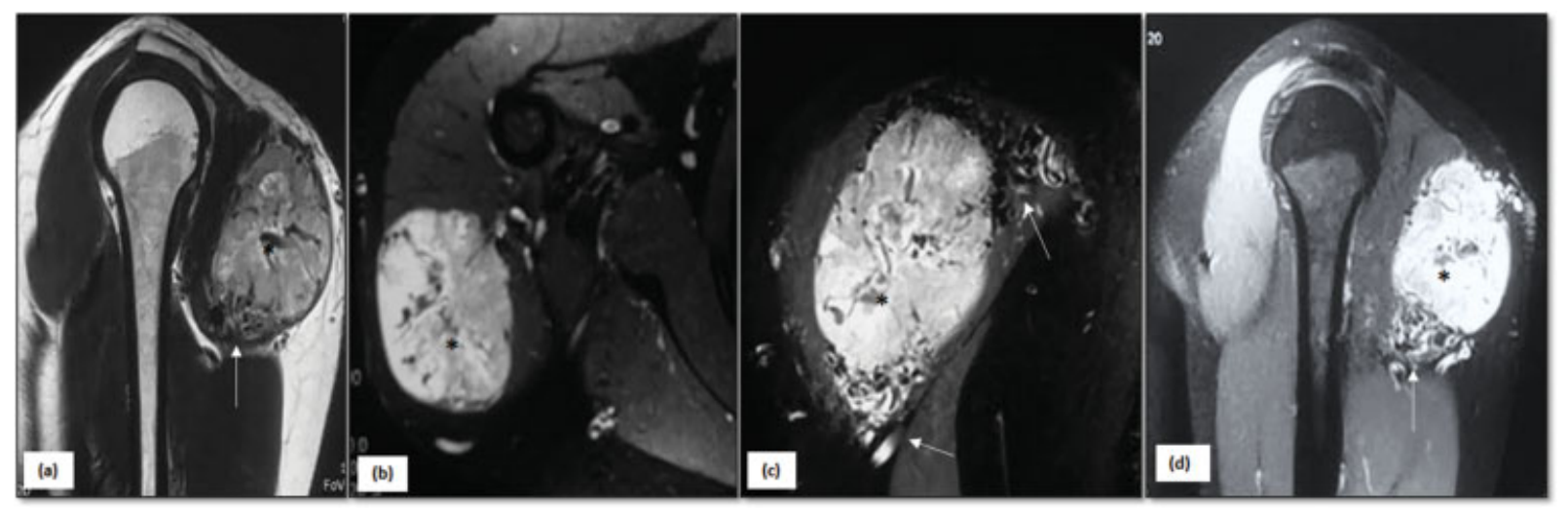

Fig. 2 ASPS of shoulder in a 17-year-old male: Sagittal T1 (a), axial (b), coronal (c), and sagittal (d) T1 PC FS MR images of right shoulder shows a well-defined mass along posterior aspect of shoulder (asterisk) infiltrating the deltoid muscle with no infiltration of neurovascular bundle or surrounding bones. The mass is hyperintense on T1 (a) as well as T2-weighted sequences and shows significant homogeneous enhancement (b-d). Multiple flow voids are seen within the mass along with tortuous feeding vessels in vicinity (arrows). ASPS, alveolar soft part sarcoma. 


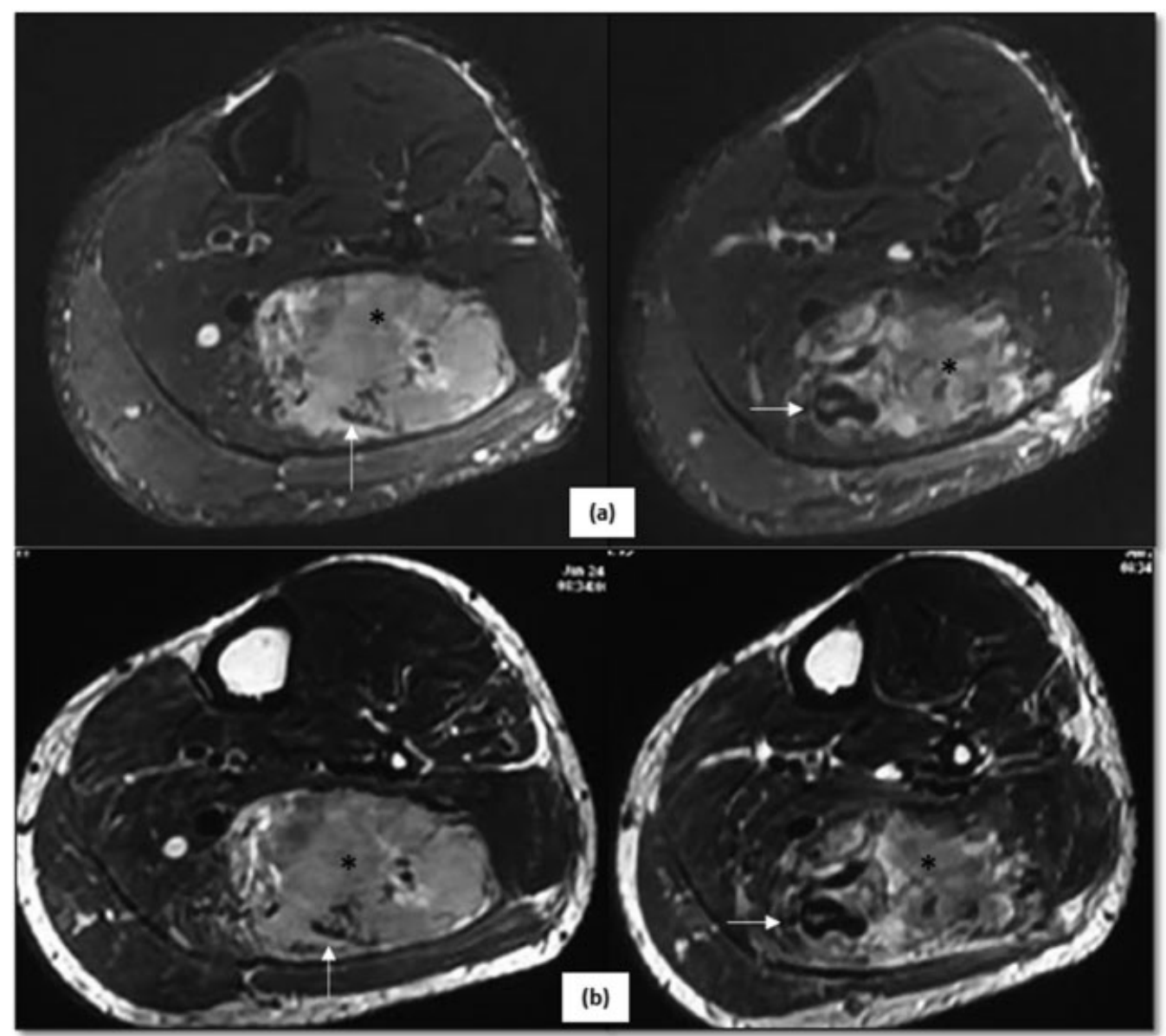

Fig. 3 ASPS in an 18-year-old male patient: Axial T2 FS (a) and axial T2 (b) MR images reveals presence of a well-defined mass (asterisk) in the posterolateral aspect of the left calf appearing hyperintense on T2WI. Multiple tortuous vessels are seen in its vicinity (arrows) and within the mass.

and scalp. Uncommon sites reported by other authors ranged from paraspinal location, tongue, larynx, and buccal space., ${ }^{43}$ Head and neck are uncommon sites, however, particularly frequently encountered in children (-Fig. 4).

Ultrasound is often done as a first-time imaging modality to evaluate when a patient presents with a soft tissue swelling which has a wide differential diagnosis ranging from developmental, inflammatory, traumatic, or neoplastic causes. Based on the clinical history and imaging appearance it is important to exclude an abscess or hematoma. Two of our patients had ultrasound available which showed a well marginated hypoechoic solid mass with multiple large vessels. Daly et al evaluated six patients of ASPS and ultrasound findings revealed a hypoechoic or mixed echotexture mass with well-defined margins and hypervascular nature with large vessels. On spectral analysis, high velocity systolic and diastolic flow was detected. $^{15}$ Ultrasound findings are not diagnostic,
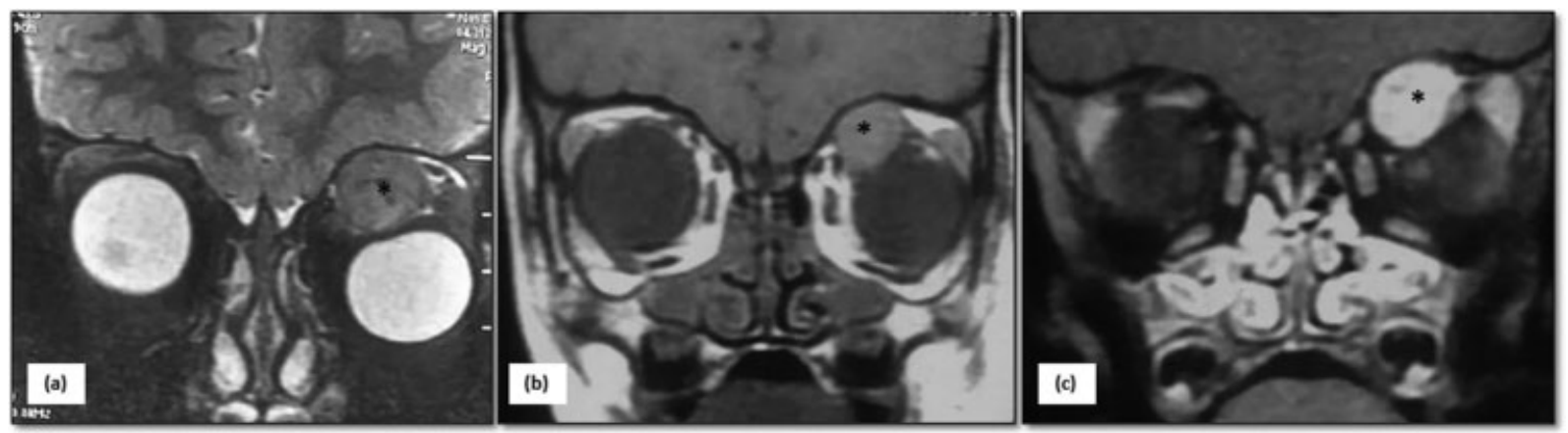

Fig. 4 ASPS of orbit in a 3-year-old boy: coronal T2 (a), T1 (b), and T1 PC (c) MRI of orbits show presence of a well-defined mass in the extraconal compartment of left orbit along the superomedial aspect (asterisk). The mass is hyperintense on T2 (a) as well as T1- (b) weighted sequence and shows homogeneous intense enhancement (c). No intracranial extension was present. ASPS, alveolar soft part sarcoma. 


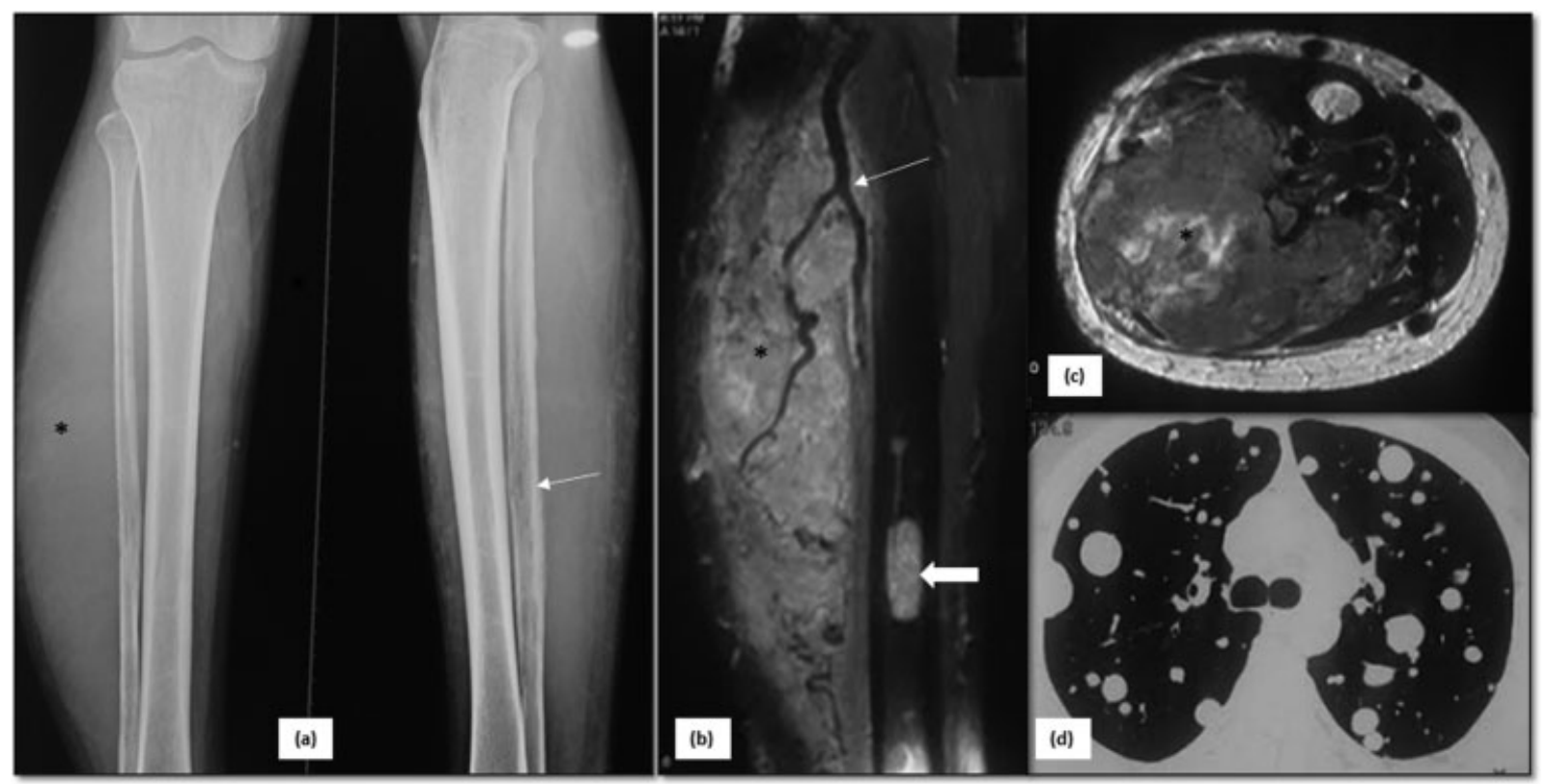

Fig. 5 ASPS of calf with lung metastases in a 29-year-old male patient: plain radiographs AP and lateral views (a) show soft tissue swelling in the region of calf along posterolateral aspect (asterisk in a) with permeative lytic changes and cortical irregularity in fibula (arrow in a). Subsequently performed MRI selected images-sagittal T2 FS (b), axial T2 (c) showed mass in the posterior aspect of calf (asterisk in b, c) with tortuous feeding vessels in the vicinity (thin arrow in b). This mass is infiltrating and eroding fibula (c) and is associated with noncontiguous metastatic lesions in tibia (thick arrow in b). Axial CT thorax (d) revealed presence of multiple metastatic lesions of variable sizes, diffusely scattered in both lung fields. ASPS, alveolar soft part sarcoma.

however, may raise the suspicion of a soft tissue tumor and prompt further imaging.

On contrast-enhanced CT and MR of the primary mass, most of the cases in our study showed well-defined lobulated margins. Well circumscribed nature of the tumor could be attributed to its indolent slow growing nature. Intense enhancement and feeder vessels were also a commonly seen feature. Intense enhancement, presence of feeders, and multiple flow voids were the most consistent findings across many studies ${ }^{4,9-12,16}$ (-Figs. 2, 3, 4, and 5). Necrosis was not seen, and calcification was only seen in one case. Presence of necrosis is related to tumor size and thus variable results were found in different case series. ${ }^{2}$
Metastases is common at the time of presentation with lung being the most frequently reported site. This is probably due to the high vascularity and early microvascular dissemination of the tumor. ${ }^{14}$ Lung was the most common site with 13 of 14 patients harboring metastatic nodules ( - Figs. 5 and $\mathbf{6}$ ). This was in keeping with findings reported by other authors. ${ }^{4}$ Other sites of metastatic disease were brain, liver, adrenal, and lymph nodes in our study. Lymph nodes is an unusual site of spread of sarcoma and angiosarcoma is the most common type to harbor nodal metastases (13.5-45\%). The same study showed the incidence of nodal spread in ASPS to be 0 to $12.5 \%{ }^{17}$ Lymph nodal metastasis was seen in only one of 16 cases in the present study. Overall metastatic disease reported in other studies

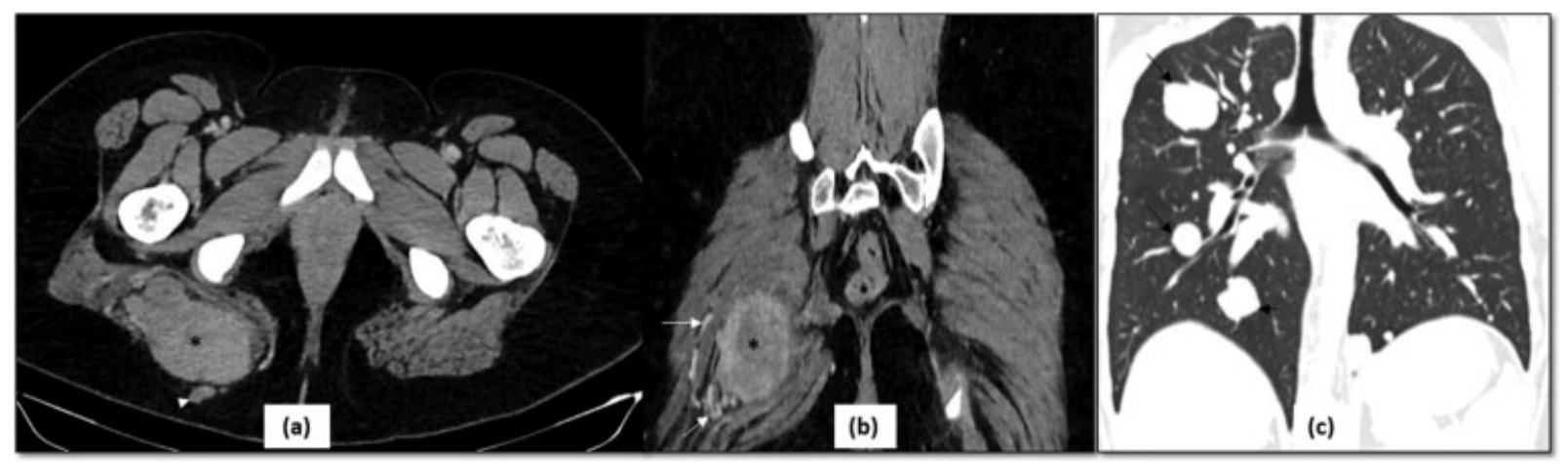

Fig. 6 ASPS in right gluteal region in a 20-year-old girl: Axial (a) and coronal (b) reformatted CT images demonstrate a circumscribed intensely enhancing homogeneous mass (asterisk) in the right gluteal region with peripheral feeding vessels (arrows in b), infiltrating the gluteal maximus muscle. A smaller satellite lesion is also seen in its vicinity in the subcutaneous plane (arrowhead in a). Though the mass appears resectable, the patient could not undergo surgery as she had lung metastases at the time of presentation, as seen on coronal (c) reformatted lung window of chest (arrows in c). ASPS, alveolar soft part sarcoma. 


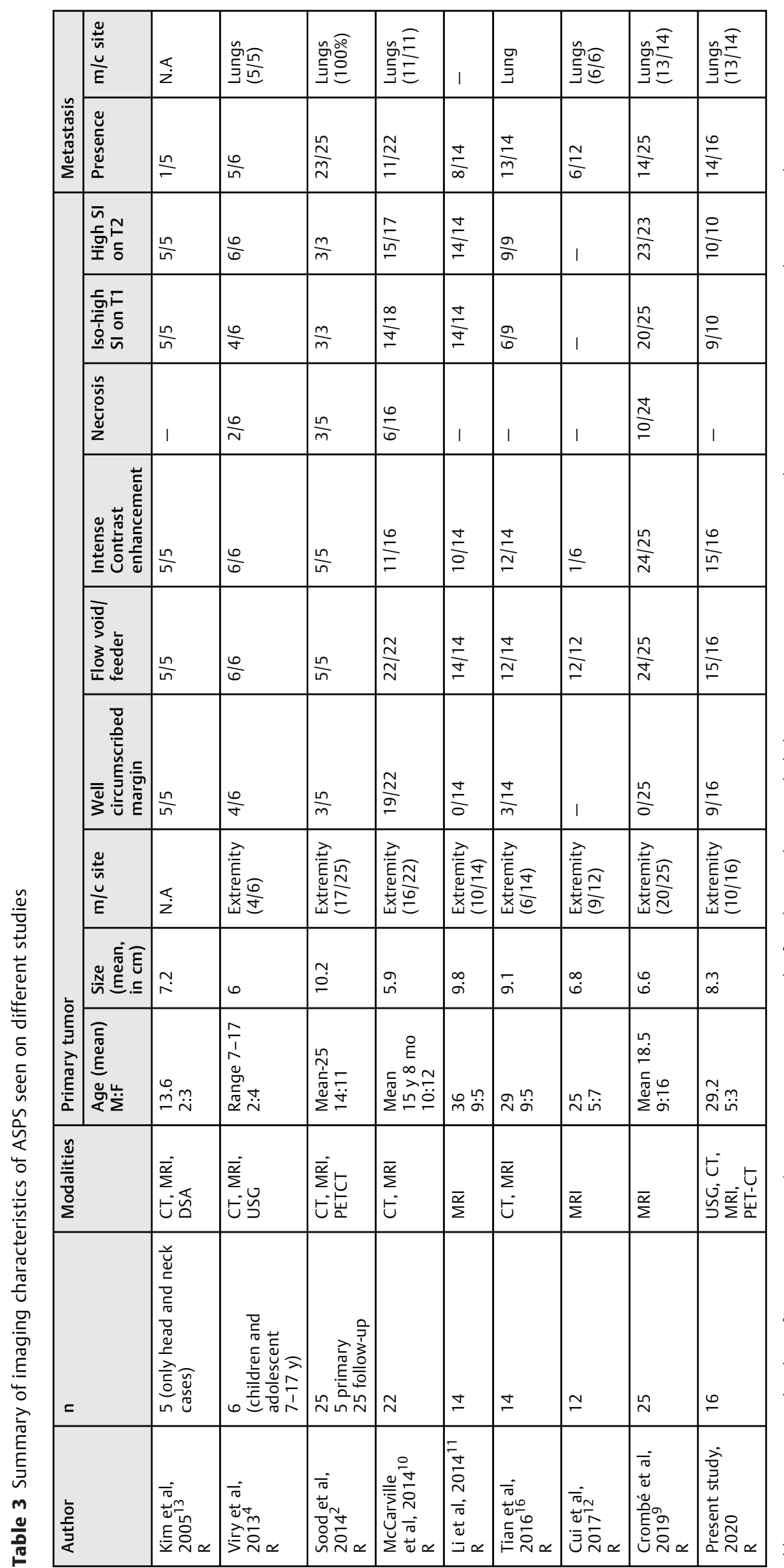


ranged from 50 to $65 \%{ }^{6,18}$ as compared with $88 \%$ in our study. This can be attributed to the fact that ours is a referral center and thus receives patients in advanced stages mostly. ASPS are indolent slow growing malignant tumors. In spite of this fact, they are usually metastatic at presentation. ${ }^{19}$ Metastatic lesions may remain stable for years on continued chemotherapy. ${ }^{20}$ Pulmonary metastatectomy is the preferred treatment in the absence of metastases at other sites. ${ }^{21}$ - Table 3 summarizes the imaging features of ASPS seen in different studies.

It is important here to discuss the various differential diagnosis of an avidly enhancing mass with feeder vessels. The possibility of a hypervascular metastasis from a renal or thyroid primary should always be kept in mind. Also, angiomatous tumors like angiosarcoma, hemangiopericytoma could also have a similar imaging appearance. ${ }^{14}$ However, these angiomatous tumors often show blood fluid levels on MRI which are not described in ASPS. ${ }^{22}$

Retrospective study design with small sample size of 16 patients due to rare nature of this sarcoma is one of the limitations of our study. Also, the imaging protocols were not standardized due to most imaging being done from outside hospitals and referred to our tertiary care center for review and further management. The imaging and histopathology were reviewed by single radiologist and pathologist from multidisciplinary sarcoma team; thus, interobserver variability was not evaluated in this study. Also, as the cohort of cases belonged to a tertiary care center, more patients with metastatic disease were seen.

In conclusion, ASPS is a rare STS seen in children and young adults having an indolent course. It should be suspected in cases showing an intensely enhancing intramuscular mass with feeder vessels. Imaging may mimic a vascular malformation or tumor due to large number of feeders. Misdiagnosis at an early stage may lead to later metastatic presentation of the disease.

Institute Ethics Obtained Vide Letter Number

IEC 527/05.06.2020

Source(s) of Support

None.

Presentation at a Meeting

None.

Conflict of Interest

None declared.

Acknowledgment

None.

\section{References}

1 American Cancer Society. Cancer Facts \& Figures 2020. Atlanta: American Cancer Society; 2020
2 Sood S, Baheti AD, Shinagare AB, et al. Imaging features of primary and metastatic alveolar soft part sarcoma: single institute experience in 25 patients. Br J Radiol 2014;87(1036):20130719

3 Kallen ME, Hornick JL. The 2020 WHO Classification: what's new in soft tissue tumor pathology? Am J Surg Pathol 2021;45(01):e1-e23

4 Viry F, Orbach D, Klijanienko J, et al. Alveolar soft part sarcomaradiologic patterns in children and adolescents. Pediatr Radiol 2013;43(09):1174-1181

5 Prasad A, Chauhan B, Pal H, Bhardwaj M. Alveolar soft part sarcoma with cerebral and skeletal metastases. Indian J Radiol Imaging 2001;11:44-45

6 Pennacchioli E, Fiore M, Collini P, et al. Alveolar soft part sarcoma: clinical presentation, treatment, and outcome in a series of 33 patients at a single institution. Ann Surg Oncol 2010;17(12): 3229-3233

7 Christopherson WM, Foote FW Jr, Stewart FW. Alveolar soft-part sarcomas; structurally characteristic tumors of uncertain histogenesis. Cancer 1952;5(01):100-111

8 Ladanyi M, Lui MY, Antonescu CR, et al. The der(17)t(X;17)(p11; q25) of human alveolar soft part sarcoma fuses the TFE3 transcription factor gene to ASPL, a novel gene at 17q25. Oncogene 2001;20(01):48-57

9 Crombé A, Brisse HJ, Ledoux P, et al. Alveolar soft-part sarcoma: can MRI help discriminating from other soft-tissue tumors? A study of the French sarcoma group. Eur Radiol 2019;29(06): 3170-3182

10 McCarville MB, Muzzafar S, Kao SC, et al. Imaging features of alveolar soft-part sarcoma: a report from Children's Oncology Group Study ARST0332. AJR Am J Roentgenol 2014;203(06):1345-1352

$11 \mathrm{Li} \mathrm{X}$, Ye Z. Magnetic resonance imaging features of alveolar soft part sarcoma: report of 14 cases. World J Surg Oncol 2014;12:36

12 Cui J-F, Chen H-S, Hao D-P, Liu J-H, Hou F, Xu W-J. Magnetic resonance features and characteristic vascular pattern of alveolar soft-part sarcoma. Oncol Res Treat 2017;40(10):580-585

$13 \mathrm{Kim}$ HS, Lee HK, Weon YC, Kim H-J. Alveolar soft-part sarcoma of the head and neck: clinical and imaging features in five cases. AJNR Am J Neuroradiol 2005;26(06):1331-1335

14 Itani M, Shabb NS, Haidar R, Khoury NJ. AIRP best cases in radiologic-pathologic correlation: alveolar soft-part sarcoma. Radiographics 2013;33(02):585-593

15 Daly BD, Cheung H, Gaines PA, Bradley MJ, Metreweli C. Imaging of alveolar soft part sarcoma. Clin Radiol 1992;46(04):253-256

16 Tian L, Cui C-Y, Lu S-Y, Cai P-Q, Xi S-Y, Fan W. Clinical presentation and CT/MRI findings of alveolar soft part sarcoma: a retrospective single-center analysis of 14 cases. Acta Radiol 2016;57(04):475-480

17 Fong Y, Coit DG, Woodruff JM, Brennan MF. Lymph node metastasis from soft tissue sarcoma in adults. Analysis of data from a prospective database of 1772 sarcoma patients. Ann Surg 1993; 217(01):72-77

18 Portera CA Jr, Ho V, Patel SR, et al. Alveolar soft part sarcoma: clinical course and patterns of metastasis in 70 patients treated at a single institution. Cancer 2001;91(03):585-591

19 Paoluzzi L, Maki RG. Diagnosis, prognosis, and treatment of alveolar soft-part sarcoma: a review. JAMA Oncol 2019;5(02):254-260

20 Read WL, Williams F. Metastatic alveolar soft part sarcoma responsive to pazopanib after progression through sunitinib and bevacizumab: two cases. Case Rep Oncol 2016;9(03):639-643

21 Kodama K, Doi O, Higashiyama M, et al. Surgery for multiple lung metastases from alveolar soft-part sarcoma. Surg Today 1997;27 (09):806-811

22 Murphey MD, Fairbairn KJ, Parman LM, Baxter KG, Parsa MB, Smith WS. From the archives of the AFIP. Musculoskeletal angiomatous lesions: radiologic-pathologic correlation. Radiographics 1995;15(04):893-917 